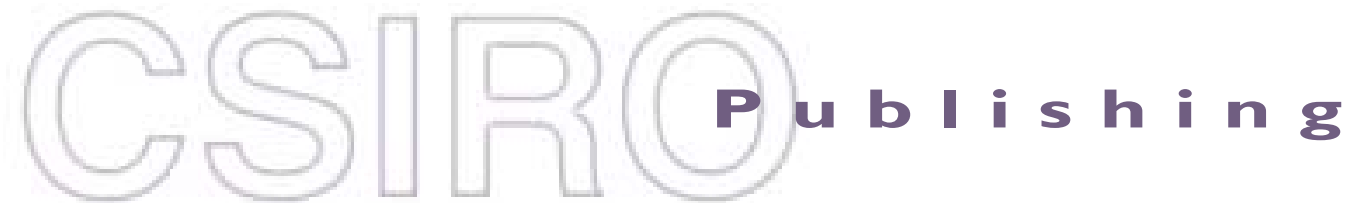

\section{Publications of the Astronomical Society of Australia}

Volume 19, 2002

(C) Astronomical Society of Australia 2002

An international journal of astronomy and astrophysics

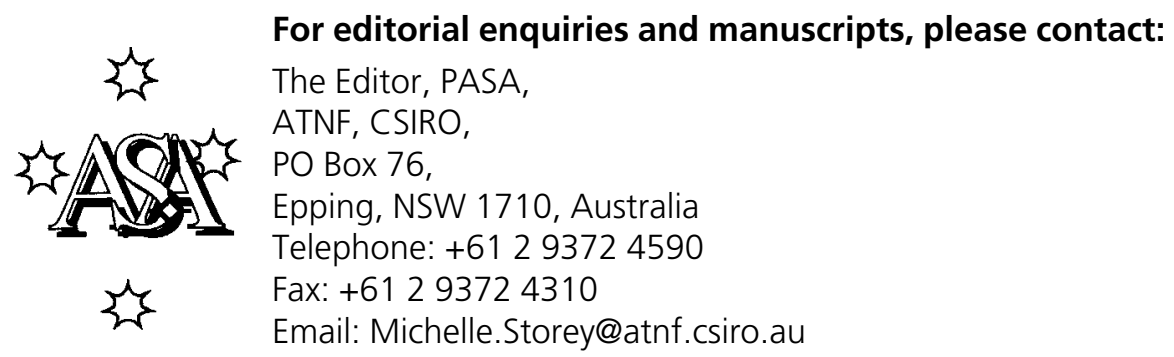

For general enquiries and subscriptions, please contact: CSIRO Publishing PO Box 1139 (150 Oxford St)

Collingwood, Vic. 3066, Australia

Telephone: +6139662 7666

Fax: +61 396627555

Email: publishing.pasa@csiro.au

C S I RO

PUBLISHING Published by CSIRO Publishing

for the Astronomical Society of Australia

www.publish.csiro.au/journals/pasa 


\title{
Operation of the Near Infrared Sky Monitor at the South Pole
}

\author{
J. S. Lawrence ${ }^{1}$, M. C. B. Ashley ${ }^{1}$, M. G. Burton ${ }^{1}$, P. G. Calisse ${ }^{1}$, J. R. Everett ${ }^{1}$, \\ R. J. Pernic ${ }^{2}$, A. Phillips ${ }^{1}$ and J. W. V. Storey ${ }^{1}$ \\ ${ }^{1}$ School of Physics, University of New South Wales, NSW 2052, Australia \\ j1@phys.unsw.edu.au \\ ${ }^{2}$ Yerkes Observatory, The University of Chicago, Williams Bay, Wisconsin 53191, USA \\ Received 2002 June 20, accepted 2002 October 3
}

\begin{abstract}
The near infrared sky spectral brightness has been measured at the South Pole with the Near Infrared Sky Monitor (NISM) throughout the 2001 winter season. The sky is found to be typically more than an order of magnitude darker than at temperate latitude sites, consistent with previous South Pole observations. Reliable robotic operation of the NISM, a low power, autonomous instrument, has been demonstrated throughout the Antarctic winter. Data analysis yields a median winter value of the $2.4 \mu \mathrm{m}$ $\left(\mathrm{K}_{\text {dark }}\right)$ sky spectral brightness of $\sim 120 \mu \mathrm{Jy} \operatorname{arcsec}^{-2}$ and an average of $210 \pm 80 \mu \mathrm{Jy} \mathrm{arcsec}^{-2}$. The $75 \%$, $50 \%$, and $25 \%$ quartile values are $270 \pm 100,155 \pm 60$, and $80 \pm 30 \mu \mathrm{Jy} \mathrm{arcsec}^{-2}$, respectively.
\end{abstract}

Keywords: atmospheric effects — radiative transfer — site testing — infrared: general

\section{Introduction}

Previous observations of the infrared sky spectral brightness at the South Pole have indicated that it is up to two orders of magnitude lower than that observed at the best temperate latitude sites. Reductions are observed in the near infrared (Nguyen et al. 1996; Ashley et al. 1996; Phillips et al. 1999) and the mid infrared (Valenziano, Cavaliere, \& Miriametro 1997; Smith \& Harper 1998; Chamberlain et al. 2000), and are predicted to also occur in the far infrared (Townes \& Melnick 1990).

The lowest infrared Antarctic sky emission will occur for wavelengths that lie on the Wien side of the Planck function (where emission is most sensitive to changes in temperature) and wavelengths for which airglow emission is lowest or non-existent. Both of these requirements are satisfied for a region of wavelengths in the atmospheric $\mathrm{K}$ window centred around $2.4 \mu \mathrm{m}$ (see for example Figure 1 of Phillips et al. 1999). Sky brightness in this window (the $K_{\text {dark }}$ window, $\sim 2.25-2.5 \mu \mathrm{m}$ ) has been utilised in many studies as a representation of site quality. From the South Pole, Nguyen et al. (1996) and Ashley et al. (1996) have reported a $K_{\text {dark }}$ sky spectral brightness of $100-200 \mu \mathrm{Jy} \operatorname{arcsec}^{-2}$, photometrically obtained with the GRIM, a near infrared grism spectrometer on the SPIREX telescope (see Hereld 1994) and the IRPS Infrared Photometer Spectrometer (see Ashley et al. 1995), respectively. Phillips et al. (1999) reported consistent values across a range of near infrared wavelengths through the 1995 South Pole winter, again with the IRPS.

The current study reports an analysis of the data collected by the Near Infrared Sky Monitor (NISM) over the 2001 season at the South Pole. The NISM, a lowpowered, autonomous, site-testing instrument, measures sky spectral brightness in the $\mathrm{K}_{\text {dark }}$ window via a differential detection technique. The mean value of the dark sky emission is determined to be $210 \mu \mathrm{Jy} \operatorname{arcsec}^{-2}$, which is consistent with the earlier studies.

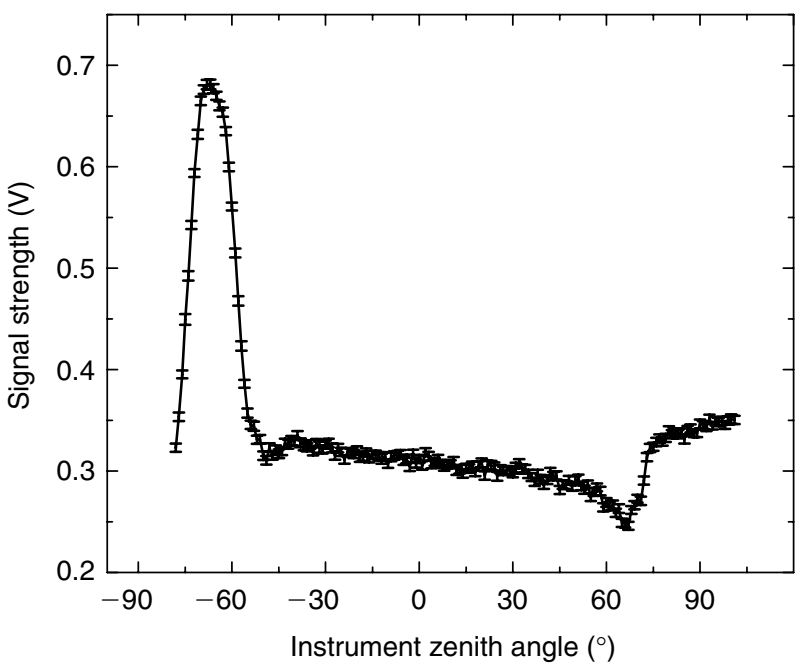

Figure 1 Example of a NISM sky dip, representing a scan of the instrument from horizon-to-horizon. The peak at $-67.5^{\circ}$ corresponds to one instrument beam pointing at the blackbody calibrator and the other at the sky.

There are several motivations for operating the NISM at the South Pole. Firstly, it is wished to measure the South Pole sky spectral brightness with a filter centre wavelength slightly longer than the IRPS or GRIM filters to further reduce airglow emission. Secondly, confirmation is required that the differential measurement technique used by the NISM gives robust results. Thirdly, reliable robotic operation of this instrument through the Antarctic winter must be demonstrated, in order that it can be deployed to more remote locations on the Antarctic Plateau (specifically Dome C) over the 2004 Antarctic winter. Finally, it is important that data from any site proposed for future large telescopes be acquired over a number of seasons, in order to estimate year-to-year variability. 


\section{Instrument Configuration}

The NISM is situated on the roof of the Automated Astrophysical Site Testing Observatory (AASTO), a facility derived from the Automatic Geophysical Observatory (AGO) design, and has been in operation at the AmundsenScott South Pole station since 1997 (for more details of the AASTO see Storey, Ashley, \& Burton 1996; Storey 1998). The NISM observes in two 4 degree field-ofview (FOV) beams angularly separated by $45^{\circ}$, which are collimated and alternately detected (via a reflective chopper wheel) with a Stirling cycle cooled single-element InSb detector. The cooled NISM filter has a centre wavelength of $2.379 \mu \mathrm{m}$, and an optical bandwidth of $0.226 \mu \mathrm{m}$ (FWHM). The detector measures the difference signal between the two beams. The instrument FOV is scanned in elevation across the sky through $180^{\circ}$ and back, every four hours. A calibration blackbody (an oxygen-free copper cavity coated with high infrared absorption paint) is positioned at one horizon.

The instrument is operated remotely and has a power budget of $\sim 10 \mathrm{~W}$. Data, which include instrumental diagnostics, temperatures of the black body calibrator, and the differential spectral brightness measurement and instrumental noise as a function of instrument zenith angle, are sent via satellite to an email account at the University of New South Wales. Control parameters include the integration period, the angular step size, and the detector gain. In the current observation regime a $1 \mathrm{~s}$ integration (corresponding to 77 samples) is taken at each elevation position (one per degree). The instrumental noise figure returned by the instrument is the standard deviation over the 77 samples. For more details of the operation of the NISM (and its companion mid infrared instrument, the MISM) see Storey et al. (1999).

The NISM has been in operation at the South Pole station since 2000 March. However, no useful data were collected over the 2000 season due to instrumental problems. Between the austral sunset (in late $2001 \mathrm{March}$ ) and 2001 May 18, the instrument was operating reliably but detector problems obscured the data in noise. Data were lost for approximately two weeks in late August due to a software problem. Apart from this period the instrument has collected reliable noise-free data until midNovember (well into the Antarctic summer) when it was turned off for maintenance. This paper presents analysis of NISM data from 2001 May 18 to September 10 (two weeks before sunrise).

A number of anomalies in the data analysis has led to the conclusion that the instrument filter was not properly blocking all out-of-band radiation. This was confirmed by spectrophotometric tests when the NISM was brought back from the South Pole to UNSW in 2002. A leakage of approximately $0.01 \%$ from 2.5 to $5 \mu \mathrm{m}$ was discovered. Because of the rapidly increasing blackbody flux at these wavelengths, even a very small amount of longwavelength leakage has a significant effect. Fortunately, although the data analysis procedure is complicated by this filter leakage, it is still possible to obtain the sky spectral brightness within the $\mathrm{K}_{\mathrm{dark}}$ band, which is the region of interest.

An example of the NISM signal is shown in Figure 1; this represents a single horizon-to-horizon scan of the instrument (a sky dip). The instrument zenith angle is the angle between the zenith and the mid-point between the two sky beams. One horizon is defined as being at $-90^{\circ}$, the zenith at $0^{\circ}$, and the other horizon at $+90^{\circ}$. A DC offset has been subtracted from the signal, and the instrument angle is calibrated to $-67.5^{\circ}$ at the peak of the blackbody measurement. The differential signal measurement is largest at this point, when one signal beam is directly facing the blackbody, and the other oriented at $-45^{\circ}$.

Over 1200 individual winter sky dips were obtained with the NISM between 2001 May 18 and September 10. Of these, approximately $40 \%$ were rejected from further analysis. Discarded data sets include: (1) any sky dip in which there are spurious signals, which may arise from unusual instrumental noise, the presence of bright stars or emission from the moon in the instrument field of view, or changing ground conditions; (2) highly asymmetric (horizon-to-horizon) sky dips or scans that are asymmetric from one scan to the next, which indicate changing sky conditions and the presence of significant cloud or blowing snow; and (3) sky dips for which the curve fits (described in the next section) generate residuals much higher than the average instrumental error, which represent conditions inappropriate to the model used. Including data from all sky dips does not give a significantly different result to excluding sky dips with the above conditions, because such sky dips predict an incorrect sky spectral brightness (that is somewhat random).

\section{Data Analysis}

The sky spectral brightness in the near infrared arises from two principal emission mechanisms: airglow and thermal emission. The airglow occurs primarily due to chemiluminescent excitation of rovibrational transitions of $\mathrm{OH}$ molecules in the upper atmosphere boundary layer between the homosphere and the heterosphere $(80-110 \mathrm{~km})$. Thermal emission arises because the troposphere radiates like an optically thin blackbody, to which an effective temperature (which is the convolved atmospheric temperature profile) can be ascribed.

The term 'sky spectral brightness' is used in the current paper, following common nomenclature in astrophysical site-testing literature, to represent a spectral flux density per unit solid angle, in units of $\mathrm{W} \mathrm{m}^{-2} \mathrm{~Hz}^{-1} \mathrm{sr}^{-1}$ (equivalent to $2.35 \times 10^{15} \mathrm{Jy}_{\operatorname{arcsec}^{-2}}$ ). Such a unit is correctly called a spectral radiance, commonly called specific intensity, although it is called brightness in the astrophysical literature.

Equations of radiative transfer for a stratified, twolayer, isotropic, plane-parallel, non-scattering atmosphere (see Figure 2) in thermodynamic equilibrium give the approximate detected signal, $S$, as a function of zenith 


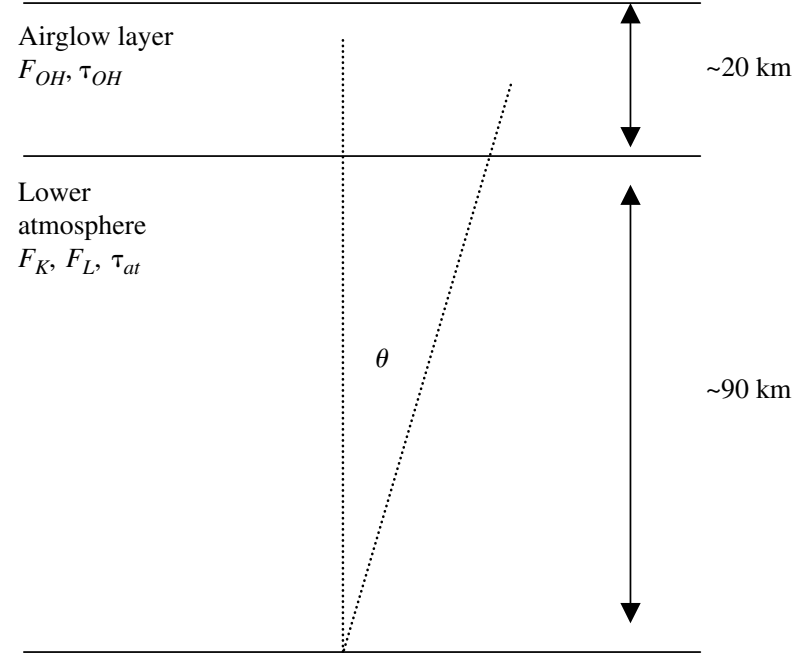

Earth

Figure 2 Schematic showing the two-layer plane parallel model used to calculate the sky spectral brightness from the radiative transfer equations. Emission, $F$, and absorption, $\tau$, occur in both the lower atmosphere (at) layer and the upper airglow layer $(\mathrm{OH})$. Lower atmosphere flux has a component in both the instrument bandpass $(K)$ and the leakage bandpass $(L)$.

angle, $\theta$, for each detected sky beam as (Jacobson 1999; Phillips et al. 1999; Chamberlain et al. 2000)

$$
\begin{aligned}
S(\theta) \approx & R\left[F_{\text {in }}+f_{K} F_{K}\left(1-e^{-\tau_{a t} \sec \theta}\right)\right. \\
& \left.+f_{K} F_{O H} \sec (\theta) e^{-\tau_{a t} \sec \theta}+F_{K}\left(1-f_{K}\right)\right]
\end{aligned}
$$

where $R$ is the instrument responsivity (including detector quantum efficiency and system optical loss), $F_{\text {in }}$ is the instrument emission, $F_{K}$ is the atmospheric thermal emission (from a blackbody at the effective temperature of the atmosphere) within the $\mathrm{K}_{\text {dark }}$ band, and $\mathrm{F}_{\mathrm{OH}}$ is the airglow emission. A term denoting the fraction of the instrument bandpass that is optically thin, $f_{K}$, appears because the tropospheric optical depth is nonuniform across the filter bandpass. Absorption within the lower atmospheric layer $K_{\text {dark }}$ band is assumed to occur uniformly, and is described by a single parameter: the optical depth, $\tau_{a t}$. Any contribution from scattering into the beam is neglected.

The effects of the filter leakage within the waveband $2.5-5 \mu \mathrm{m}$ are modelled by including a component due to the atmospheric thermal emission within this leakage waveband, $F_{L}$, an associated optically thin component of this waveband, $f_{L}$, and a fractional leakage of the filter, $L$,

$$
\begin{aligned}
S(\theta) \approx & R\left[F_{\text {in }}+f_{K} F_{K}\left(1-e^{-\tau_{a t} \sec \theta}\right)\right. \\
& +f_{K} F_{O H} \sec (\theta) e^{-\tau_{a t} \sec \theta}+F_{K}\left(1-f_{K}\right) \\
& \left.+f_{L} L F_{L}\left(1-e^{-3 \tau_{a t} \sec \theta}\right)+L F_{L}\left(1-f_{L}\right)\right] .
\end{aligned}
$$

In the following analysis, it is shown that despite the presence of this leakage it is still possible to obtain robust values of the $\mathrm{K}_{\mathrm{dark}}$ sky spectral brightness. The optical depth of the optically thin component of the atmosphere is assumed to be uniform throughout the leakage window
$(2.5-5 \mu \mathrm{m})$. The atmospheric transmission throughout this window has been examined with an ATRAN atmospheric model. The integrated optical depth throughout the leakage window is found to be approximately three times the integrated optical depth in the NISM filter window for a range of atmospheric water vapour content. The leakage window optical depth is thus assumed to be $3 \tau_{a t}$.

The above approximation (equation 2) is valid provided that the optical depth within the airglow layer, $\tau_{\mathrm{OH}}$, is negligible. The optical depth at the centre frequency, $v$, of an emission line of width $\Delta v$ emitted in a homogenous and isotropic media is given by (Lang 1980)

$$
\tau_{O H}=\frac{c^{2}}{8 \pi v^{2}} \frac{1}{\Delta v}\left[\exp \left(\frac{h v}{k T}\right)-1\right] A_{12} N_{L}
$$

where $N_{L}$ is the column density of excited state molecules. Assuming a mesopause temperature, $T=150 \mathrm{~K}$, an $\mathrm{OH}$ concentration of $1 \times 10^{12}$ molecules $\mathrm{m}^{-3}$ (McKewon \& Phillips 1975), and typical values for the Doppler broadened linewidth $(\Delta v=100 \mathrm{kHz})$, and Einstein coefficient $\left(A_{12}=0.00168 \mathrm{~s}^{-1}\right)$, computed from the HITRAN molecular data base (Rothman et al. 1998), give an $\mathrm{OH}$ emission line optical depth of $<1 \times 10^{-7}$. This is for the strongest of the $162 \mathrm{OH}$ emission lines within the NISM filter bandwidth; the approximation $\tau_{\mathrm{OH}} \ll 1$ therefore holds and equation (2) needs no correction for this effect.

The NISM makes a purely differential spectral brightness measurement; the blackbody calibrator temperature is then used to give a measure of the zenith sky spectral brightness. Note that any instrument emission, $F_{\text {in }}$, automatically cancels out in this analysis. The signal strengths at two instrument positions: $\alpha$, which corresponds to one instrument arm pointing at zenith and the other at $45^{\circ}$ (i.e. $\theta= \pm 67.5^{\circ}$ ), and $\beta$, which corresponds to one arm pointing at $45^{\circ}$ and the other at the blackbody angle $\left(\theta= \pm 22.5^{\circ}\right)$, are used. Their ratio,

$$
\frac{\alpha}{\beta}=\frac{R[S(45)-S(B B)]}{R[S(0)-S(45)]},
$$

can be used to derive the zenith sky spectral brightness,

$$
F^{K+L}(0)=\left(F_{b b K}+L F_{b b L}\right)\left[X+\frac{\alpha}{\beta} X-\frac{\alpha}{\beta}\right]^{-1},
$$

where the superscript $(K+L)$ refers to the fact that the spectral brightness determined has contributions from both the $\mathrm{K}_{\text {dark }}$ window and the leakage window. The brightness of the calibration blackbody source $\left(F_{b b K}+\right.$ $\left.L F_{b b L}\right)$ is determined from the sum of the Planck function at the temperature of the blackbody, integrated over both the filter bandwidth and the leakage bandwidth. This temperature is measured with a platinum resistance thermometer. $X$ is the ratio of the spectral brightness at $45^{\circ}$ to 
the spectral brightness at zenith (from equation 2):

$$
\begin{aligned}
X & =\frac{F(45)-F_{I N}}{F(0)-F_{I N}} \\
& =\frac{d_{1}+1-f_{K} e^{-\sqrt{2} \tau_{a t}}\left(1-\sqrt{2} d_{2}\right)-f_{L} d_{1} e^{-3 \sqrt{2} \tau_{a t}}}{d_{1}+1-f_{K} e^{-\tau_{a t}}\left(1-d_{2}\right)-f_{L} d_{1} e^{-3 \tau_{a t}}}
\end{aligned}
$$

where

$$
\begin{aligned}
& d_{1}=\frac{L F_{L}}{F_{K}}, \\
& d_{2}=\frac{F_{O H}}{F_{K}} .
\end{aligned}
$$

To determine the sky spectral brightness thus requires estimates of the atmospheric optical depth, the ratio of airglow flux to thermal atmospheric flux, the ratio of the leakage flux to the $\mathrm{K}_{\text {dark }}$ flux, and the optically thin component of both the $\mathrm{K}_{\mathrm{dark}}$ bandpass and the leakage bandpass. The first two of these parameters can be determined from curve fits to the NISM skydip data.

The instrument differential spectral brightness measurement as a function of instrument angle can be described by

$$
\begin{aligned}
\Delta S(\theta)=R[ & S(\theta+22.5)-S(\theta-22.5)] \\
=d_{3}[ & \left\{e^{-\tau_{a t} \sec (\theta-22.5)}-e^{-\tau_{a t} \sec (\theta+22.5)}\right\} \\
& +d_{4}\left\{e^{-3 \tau_{a t} \sec (\theta-22.5)}-e^{-3 \tau_{a t} \sec (\theta+22.5)}\right\} \\
& +d_{2}\left\{\sec (\theta+22.5) e^{-\tau_{a t} \sec (\theta+22.5)}\right. \\
& \left.\left.-\sec (\theta-22.5) e^{-\tau_{a t} \sec (\theta-22.5)}\right\}\right]
\end{aligned}
$$

where

$$
\begin{gathered}
d_{3}=R\left(f_{K} F_{K}\right), \\
d_{4}=\frac{f_{L} L F_{L}}{f_{K} F_{K}} .
\end{gathered}
$$

Curve fits yield the parameters $\tau_{a t}, d_{2}, d_{3}$, and $d_{4}$. A consequence of the differential measurement is that the instrumental responsivity, atmospheric thermal emission, and the fractions of the optically thin bandpass cannot be separately determined.

Histograms of curve-fitted optical depth values are shown in Figure 3. These show a mean optical depth of $\sim 0.4$, with a median value of $\sim 0.2$. The optical depth values are consistent with the dark sky values determined with the IRPS instrument, 0.1-0.3 (Phillips et al. 1999).

A histogram of the curve-fitted parameter $d_{2}$, which represents the ratio of the airglow emission to thermal emission, is shown in Figure 4. For more than $70 \%$ of the time, the airglow emission is less than $2 \%$ of the value of the thermal emission. This agrees with expectations based on the fact that there are only four strong $\mathrm{OH}$ transitions in the NISM filter window, and these are more than two orders of magnitude lower in line strength than the hundreds of strong $\mathrm{OH}$ lines observed at shorter infrared wavelengths where airglow emission dominates the night sky. Additionally, the $\mathrm{K}_{\mathrm{dark}}$ bandpass occurs at

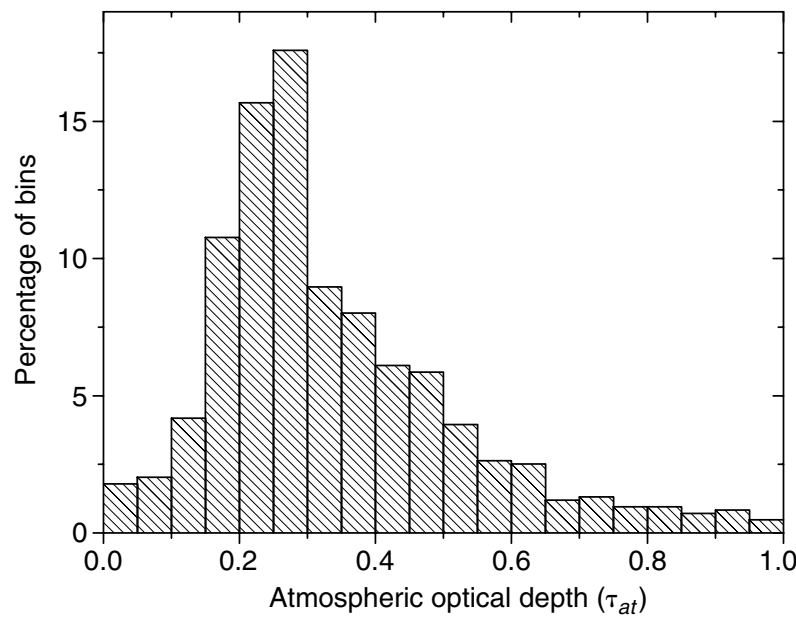

Figure 3 Histogram of optical depth values determined from curve fits of NISM sky dips to equation (8).

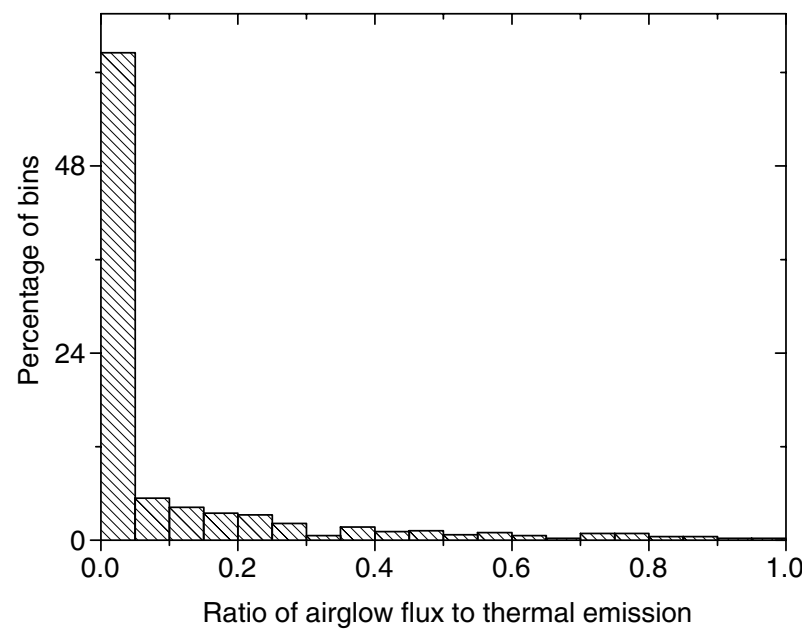

Figure 4 Histogram of values of $d_{2}$, representing the ratio of the airglow emission to the thermal emission, determined from curve fits to NISM sky dips to equation (8).

the point at which thermal emission is rising with the highest gradient. However, curve fits show that under certain atmospheric conditions the airglow flux can contribute as much as the thermal flux. This is also consistent with expectations because the concentration of $\mathrm{OH}$ within the upper tropopause is significantly variable over time and strongly depends on its position above the Earth's surface and the atmospheric conditions in the lower atmosphere (McKewon \& Phillips 1975).

Analysis with the HITRAN atmospheric molecular database (Rothman et al. 1998) indicates that over 10000 molecular lines exist within the NISM filter window. Neglecting line strengths that are more than four orders of magnitude below the highest leaves $\sim 3000$ molecular absorption lines. The ratio of the FWHM of those lines that fall within the filter window, calculated at an average atmospheric pressure and temperature, to the filter optical bandpass is $\sim 0.05$. This represents an upper limit to the optically thick component within the filter window as many of the observed lines are very weakly absorbing, 


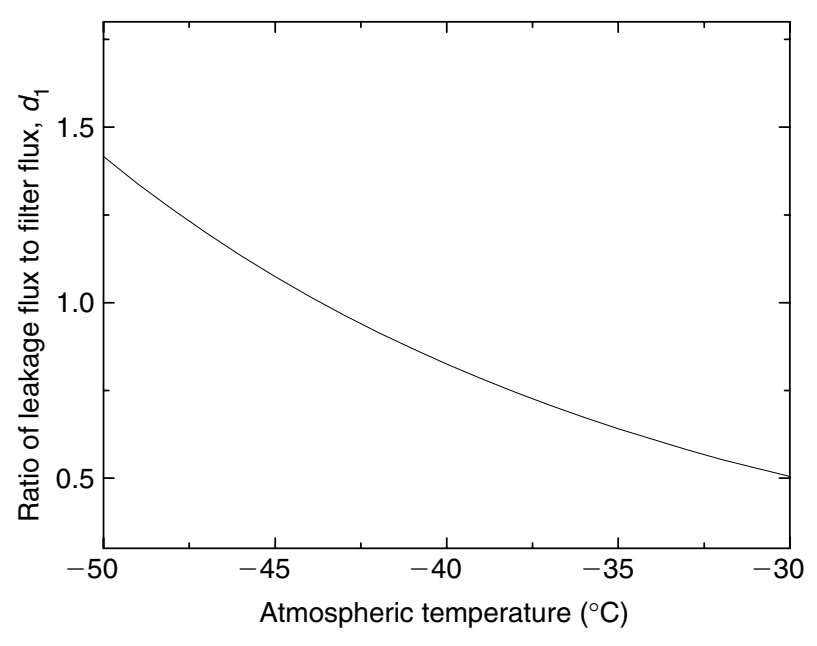

Figure 5 Ratio of the blackbody brightness integrated over the leakage band to the blackbody brightness integrated over the filter window, versus temperature. The leakage factor $L=0.01 \%$ is assumed uniform across the leakage window.

or absorb strongly only under some atmospheric conditions. Thus $f_{K}$ is assigned the value 0.95 . Such a value is in agreement with the IRPS sky dip analysis, which, being a calibrated photometric measurement, could fit the (angle independent) DC sky term. For the similar filter with which IRPS was equipped, the sky DC component contribution was determined to be 0.04 , comprising both instrument and optically thick transmission.

Similar examination of the atmospheric transmission within the leakage bandpass $(2.5-5 \mu \mathrm{m})$ can be used to determine the fraction of the optically thin component $f_{L}$ of this window. Throughout this region the atmosphere is mostly transparent, except for two optically thick regions at 2.6-2.8 $\mu \mathrm{m}$ and $4.2-4.5 \mu \mathrm{m}$, and a series of narrow lines around $3.3 \mu \mathrm{m}$. Thus $f_{L}$ is given the value 0.75 .

Figure 5 shows the ratio of the blackbody flux integrated over the leakage bandpass to the blackbody flux integrated over the filter bandpass for a range of atmospheric temperatures, assuming that the leakage is $0.01 \%$ throughout the leakage bandpass. From this, the ratio of leakage flux to filter flux, $d_{1}$, is determined. At an atmospheric temperature of $-40^{\circ} \mathrm{C}$, the contribution of flux within the leakage bandpass to that within the filter bandpass is approximately equal. Neither term exceeds the other by more than a factor of two for temperatures between $-30^{\circ} \mathrm{C}$ and $-50^{\circ} \mathrm{C}$. The ratio $d_{1}$ is thus set to 1 .

Substitution of the values for optical depth $\tau_{a t}$ and airglow emission ratio $d_{2}$ from the curve fits, the estimated parameters $f_{K}, f_{L}, d_{1}$, and the blackbody flux calculated from the blackbody temperature into equation (4) gives the total (i.e. over the whole instrument bandpass including the leakage flux) zenith sky spectral brightness $F^{K+L}(0)$. However, the sky spectral brightness of interest is the component in the $\mathrm{K}_{\text {dark }}$ window, $F^{K}(0)$. Neglecting airglow emission, the total flux is given (from equation (1)) by

$$
\begin{aligned}
F^{K+L}(0) & =F^{K}(0)+F^{L}(0) \\
& =F_{K}\left(1-f_{K} e^{-\tau}\right)+F_{L}\left(1-f_{L} e^{-3 \tau}\right)
\end{aligned}
$$

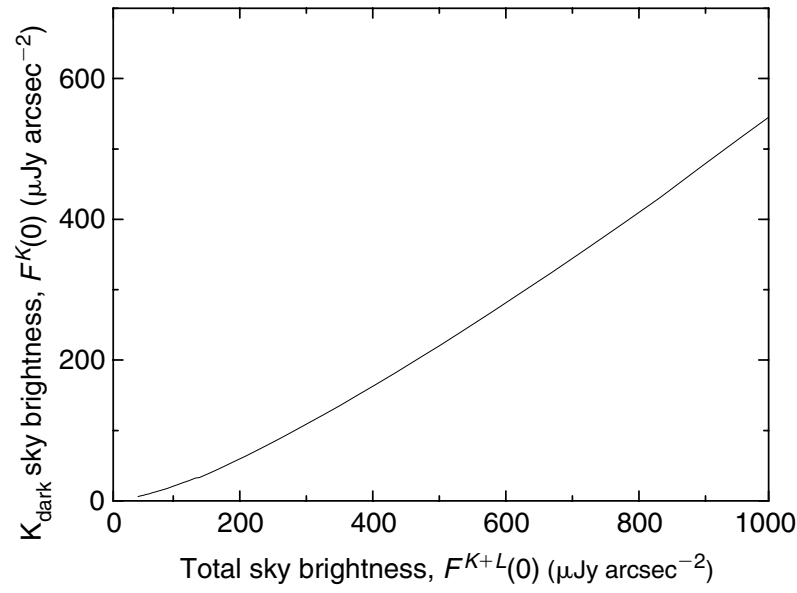

Figure $6 \mathrm{~K}_{\text {dark }}$ sky spectral brightness corrected for leakage versus raw spectral brightness for an atmospheric optical depth of 0.3 . Temperatures range from $-30^{\circ} \mathrm{C}$ to $-50^{\circ} \mathrm{C}$.

Numerical simulations of this equation for a range of atmospheric temperatures between $-30^{\circ} \mathrm{C}$ and $-50^{\circ} \mathrm{C}$ give a relationship between the total sky spectral brightness, $F^{K+L}(0)$, and the $\mathrm{K}_{\mathrm{dark}}$ spectral brightness, $F^{K}(0)$, for given values of $f_{K}, f_{L}$, and $\tau_{a t}$. This relationship does not change significantly with optical depth, and is shown in Figure 6. A fourth order polynomial gives a good fit to this function. Substitution of the total sky spectral brightness derived from equation (5) into

$$
\begin{aligned}
F^{K}(0)= & a_{0}+a_{1} F^{K+L}(0)+a_{2} F^{K+L}(0)^{2} \\
& +a_{3} F^{K+L}(0)^{3}+a_{4} F^{K+L}(0)^{4}
\end{aligned}
$$

then gives the $K_{\text {dark }}$ sky spectral brightness, where $a_{0}, \ldots, a_{4}$ are the coefficients of the polynomial fit.

\section{Results and Discussion}

The $\mathrm{K}_{\mathrm{dark}}$ sky spectral brightness as a function of time (from 2001 May 18 to September 10), shown in Figure 7, is calculated from equation (13) with assumptions and approximations as discussed in the previous section. Also shown in the graph is the cloud cover (in eighths) from South Pole meteorological records.

The sky spectral brightness consistently drops below $200 \mu \mathrm{Jy} \operatorname{arcsec}^{-2}$, and is generally stable over several hours, showing trends determined by slowly varying atmospheric conditions that can last several days. Intermittent periods of very strong atmospheric emission $\left(\sim 1000 \mu \mathrm{Jy}_{\operatorname{arcsec}^{-2}}\right)$ are also observed. These high brightness values correspond well with periods of high cloud cover (which represent a high optical depth), although the cloud-cover statistics are daily approximations from subjective observations only. A histogram of winter sky spectral brightness is shown in Figure 8. The mean winter value is $210 \mu \mathrm{Jy} \operatorname{arcsec}^{-2}$. The average values for the best $75 \%, 50 \%$, and $25 \%$ of the time are 135 , 100 , and $72 \mu \mathrm{Jy} \mathrm{arcsec}^{-2}$ respectively, and the $75 \%, 50 \%$, and $25 \%$ quartile values are 270,155 , and $80 \mu \mathrm{Jy} \operatorname{arcsec}^{-2}$ respectively. 


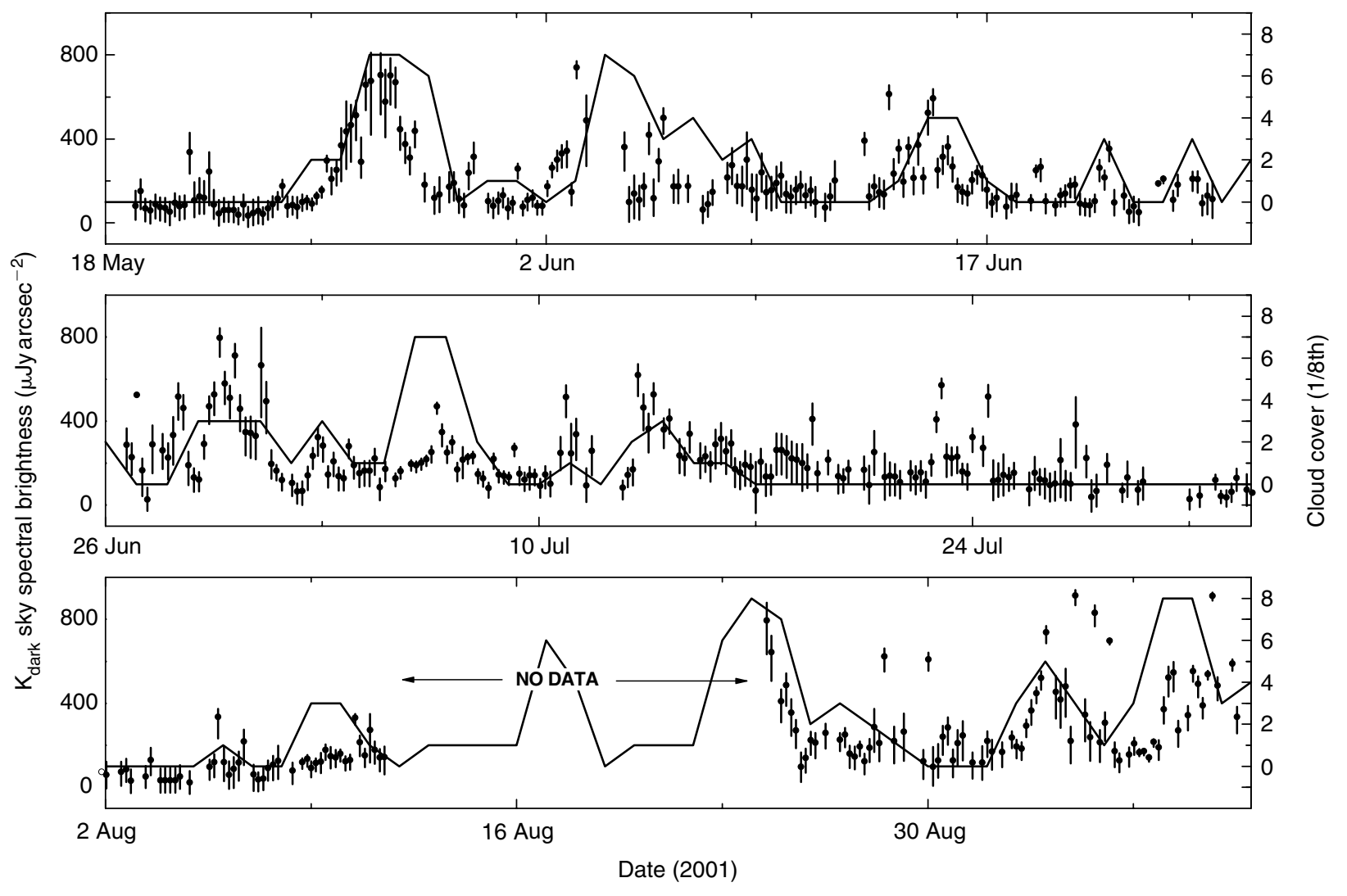

Figure 7 Dots are sky spectral brightness calculated from equation (12) over the period 2001 May 18 to September 10. The error bars represent instrumental detector noise. The line is cloud cover (in eighths) from South Pole meteorological records.

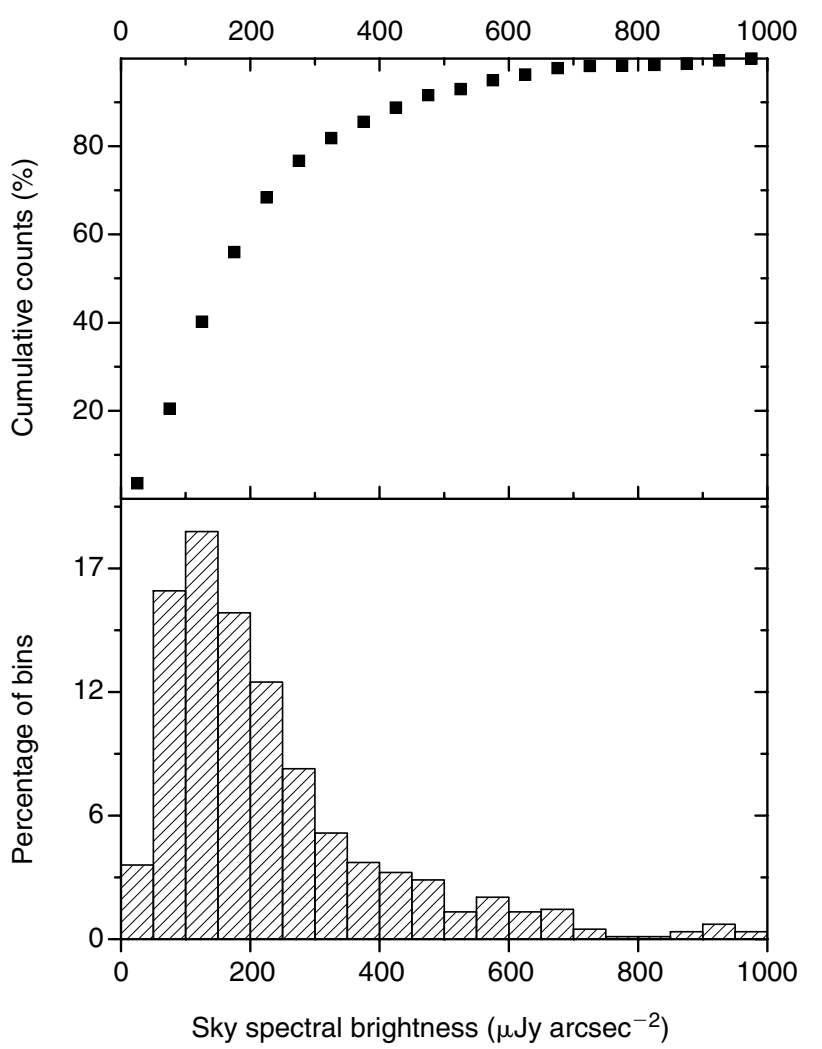

Figure 8 Histogram of sky spectral brightness values calculated from equation (12) as shown in Figure 7.
The maximum error that could result from correcting for the long-wavelength filter leakage and from plausible errors in the assumptions used to derive the sky spectral brightness has been calculated. Error is introduced in the determination of the sky spectral brightness through each of the estimated or calculated parameters. The sky spectral brightness has been calculated for variations in each of these parameters. If the error in the optical depth determined from curve fitting of the NISM sky dip data is assumed to be $10 \%$, this leads to a $\sim 4 \%$ error in the determined sky spectral brightness (with high optical depth values corresponding to high spectral brightness). An error of $50 \%$ in the value of $d_{1}$ (i.e. $d_{1}=0.5-1.5$ ) results in an error of $\sim 8 \%$ in the sky spectral brightness. A $6 \%$ error in the value $f_{K}$ (i.e. $f_{K}=0.9-1.0$ ) leads to only a $\sim 2 \%$ error in the sky spectral brightness, while an error of $6 \%$ in $f_{L}\left(f_{L}=0.7-0.8\right)$ leads to a $\sim 14 \%$ error in the sky spectral brightness. A $50 \%$ error in the value of the leakage fraction $L$ (i.e. $L=0.005-0.015 \%$ ) gives a final error of $\sim 4 \%$ (with higher leakage fraction resulting in a lower sky spectral brightness). The total possible error introduced by these assumptions is thus $\sim 30 \%$, which is comparable with the statistical error of $\sim 25 \%$. The total error is thus $\sim 40 \%$.

The data show a strong increase in sky brightness from the beginning of September. This is due to an increase in scattered sunlight at high atmospheric altitudes; the sun begins to rise at the South Pole on $\sim$ September 18 . 

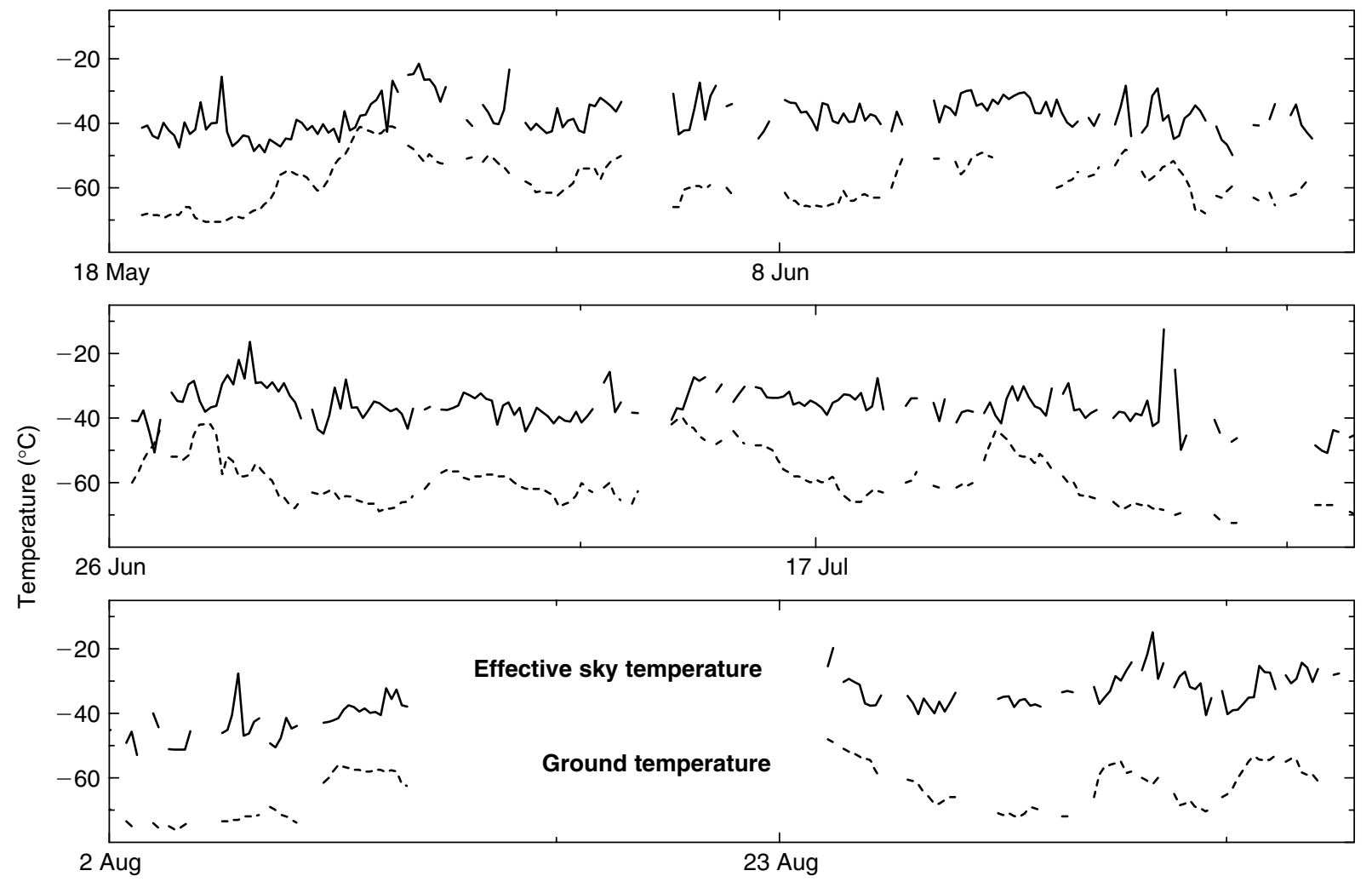

Date (2001)

Figure 9 Effective sky temperature (upper solid curve), calculated from the flux and the derived optical depth (from curve fits to the NISM data), and ground temperature (lower dashed curve) from South Pole meteorological records.

Analysis of data taken after this time is unreliable as the strong signal of the scattered sunlight interferes with the blackbody calibration and introduces spurious signals to the sky dip that depend on the time of day, position of the sun, and instrument angle, making curve fitting difficult.

From the calculated sky spectral brightness, an estimate of the effective atmospheric temperature can be made by substituting the derived values of $\mathrm{K}_{\text {dark }}$ spectral brightness and optical depth into equation (1) and calculating the temperature from the value $F_{K}$ and the Planck blackbody function, assuming airglow emission is negligible. The effective atmospheric temperature is plotted over time in Figure 9. The ground temperature obtained from South Pole meteorological records is also shown (CMDL 2001). The atmospheric temperature derived in this way has an average value of $-40^{\circ} \mathrm{C}$ and is generally $\sim 10-30^{\circ} \mathrm{C}$ higher than the ground temperature (except for periods of high cloud cover where the two temperatures are equal). This is exactly as expected, given the strong wintertime temperature inversion on the Antarctic plateau, and gives confidence that the derived values of sky brightness are correct. Some ground temperature trends are also qualitatively reproduced.

The NISM data can be compared to other observations of the near infrared sky spectral brightness. These other observations, which have similar centre wavelengths and optical bandwidths and are taken at a number of different locations with different detection methods, are presented in Table 1. It is difficult to be quantitative in these comparisons, primarily because of the slightly different centre frequencies and bandwidths.

The NISM has observed similar values to the IRPS and SPIREX experiments. From the filter bandpasses it is expected that the NISM should have a factor of two lower airglow emission (from the summed transition line intensities) and a factor of four higher thermal emission (from the integrated blackbody flux) than the IRPS experiment. This also implies that thermal emission is the dominant emission mechanism in the NISM wavelength interval.

Comparisons with other ground-based sites are difficult due to the NISM wavelength filter, which is not commonly used at temperate sites such as Mauna Kea or Chile. Observations with standard $\mathrm{K}$ window filters at Mauna Kea give typical sky spectral brightness values of $4000 \mu \mathrm{Jy} \operatorname{arcsec}^{-2}$ for the darkest sky conditions. Rebinning of narrow resolution sky spectra gives a rough estimate of the Mauna Kea spectral brightness at $\mathrm{K}_{\text {dark }}$ of $\sim 1000-2000 \mu \mathrm{Jy} \operatorname{arcsec}^{-2}$, which is $10-40$ times the brightness of the best South Pole conditions.

Balloon-borne observations of the near infrared sky spectral brightness taken at different locations give values that differ by a significant amount. This fluctuation is consistent with the highly variable sky brightness measurements observed by the current experiment (and Nguyen et al. 1996) due to variable atmospheric conditions. Additionally, it indicates that there is a large degree 
Table 1. Comparison of sky spectral brightness values in the $K_{\text {dark }}$ window from a number of different observation sites

\begin{tabular}{lllccl}
\hline Site & Instrument & $\begin{array}{c}\lambda \\
(\mu \mathrm{m})\end{array}$ & $\begin{array}{c}\Delta \lambda \\
(\mu \mathrm{m})\end{array}$ & $\begin{array}{c}\text { Sky Brightness } \\
\left(\mu \mathrm{Jy} \operatorname{arcsec}^{-2}\right)\end{array}$ & Reference \\
\hline Satellite & IRTS & 2.3 & 0.13 & 3 & Matsumoto et al. 1996 \\
Rocket & S-520-11 & 2.39 & 0.13 & 5 & Noda et al. 1992 \\
Rocket & K-9M-77 & 2.52 & 0.46 & 8 & Matsumoto et al. 1988 \\
Rocket & S-520-15 & 2.35 & 0.13 & 8 & Matsuura et al. 1994 \\
Balloon 32 km & Thisbe & 2.40 & 0.10 & 26 & Hoffman et al. 1977 \\
Balloon 40 km & TRIP & 2.39 & 0.12 & 35 & Mandolesi et al. 1998 \\
Balloon 40 km & & 2.38 & 0.08 & 130 & Matsumoto et al. 1994 \\
South Pole & GRIM & 2.36 & 0.14 & 162 & Nguyen et al. 1996 \\
South Pole & IRPS & 2.36 & 0.14 & 180 & Ashley et al. 1996 \\
South Pole & NISM & 2.38 & 0.23 & $210(72)^{a}$ & This work \\
South Pole & IRPS & 2.32 & 0.19 & $240(130)^{b}$ & Phillips et al. 1999 \\
Mauna Kea & & 2.22 & 0.39 & 4000 & Leihert et al. 1998 \\
Siding Spring & IRPS & 2.35 & 0.50 & 6000 & Ashley et al. 1996 \\
\hline
\end{tabular}

${ }^{a}$ Value in parentheses represents darkest $25 \%$ of dark sky values.

${ }^{b}$ Value in parentheses represents darkest $20 \%$ of dark sky values.

of variability between different sites, possibly due to different atmospheric constituent abundances, and thermal and pressure gradients. NISM values are 1-5 times larger than such balloon measurements; however, the NISM filter has twice the bandwidth. Information about the relative values of the thermal emission and airglow emission could be obtained from comparisons between ground and balloon-borne observations, but only for exactly the same sky conditions, filter bandwidths, and locations.

Rocket and satellite observations for similar wavelength and bandwidth filters give a good indication of the solar system zodiacal emission. The zodiacal emission results from sunlight scattered off interplanetary dust, and represents the limiting background radiation for any infrared astrophysical observations made from Earth or from a near-Earth orbit. Values obtained range from 3 to $8 \mu \mathrm{Jy} \operatorname{arcsec}^{-2}$, but again are for bandwidths approximately half that of the NISM filter. The South Pole infrared sky background for the darkest sky conditions is only an order of magnitude above this limit, and represents the lowest value anywhere on Earth for near infrared observations.

Although the $\mathrm{K}_{\text {dark }}$ window is more commonly used as a measure of site quality than for astrophysical observations, there are several important astronomical studies that could be done in such a window at a site with the very low background levels encountered in Antarctica. For example, the $\mathrm{v}=1-0 \mathrm{Q}$ branch $\mathrm{H}_{2}$ emission lines at 2.40-2.42 $\mu \mathrm{m}$ (resulting from either shocks or UV fluorescence) are a useful tool for studies of star formation and galactic structure. The $\mathrm{H} \alpha$ line is redshifted into the $\mathrm{K}_{\text {dark }}$ window at $\mathrm{z}=3$, and the $\mathrm{Pa} \alpha$ line at $\mathrm{z}=0.2-0.3$. Such observations are useful for extragalactic star formation studies. Searches for gravitational micro-lensing events could also benefit from the increased sensitivity in this wavelength interval combined with the possibility of continuous observation. Science goals for infrared instruments located at an Antarctic site are discussed in more detail by Burton, Storey, \& Ashley (2001). Additionally, the fact that very low sky background levels are observed in this particular window indicates that lower backgrounds should be observed throughout the infrared, and that the Antarctic plateau provides the highest infrared sensitivities per telescope area on the surface of the planet.

\section{Conclusion}

The sky spectral brightness in the near infrared $\mathrm{K}_{\text {dark }}$ window at the South Pole has been determined from analysis of data collected by the Near Infrared Sky Monitor over the period 2001 May 18 to September 10. It is found that the average wintertime sky flux is $210 \pm 80 \mu \mathrm{Jy} \mathrm{arcsec}{ }^{-2}$, and the median is $\sim 120 \mu \mathrm{Jy} \operatorname{arcsec}^{-2}$. The $75 \%, 50 \%$, and $25 \%$ quartile values are $270 \pm 100,155 \pm 60$, and $80 \pm$ $30 \mu \mathrm{Jy} \operatorname{arcsec}^{-2}$, respectively. These values compare well with those from similar bandwidth filters operated previously on other instruments at the South Pole. The data further support the proposition that the Antarctic plateau is the best site on Earth to perform near infrared astronomy. The planned move of the NISM to Dome C on the Antarctic plateau in 2004 will further quantify the advantages obtainable to the astrophysical community by further developing the Antarctic plateau as an observatory site.

\section{Acknowledgements}

The authors would like to thank the US Center for Astrophysical Research in Antarctica (CARA) for support and collaboration throughout our site testing campaign. In particular, CARA winterovers Charles Kaminski, Jan Davidson, and the late Rodney Marks provided invaluable support for the winter operation of the NISM. Logistic support by the US National Science Foundation (NSF) is gratefully acknowledged. We thank Alan Tokunaga for 
drawing to our attention the possibility that long wavelength leakage could explain our data anomalies, and the anonymous referee who encouraged us to make an even more rigorous analysis of this issue. This research has been funded by the Australian Research Council and by an Australian Antarctic Division AAS grant.

\section{References}

Ashley, M. C. B., Burton, M. G., Lloyd, J. P., \& Storey, J. W. V. 1995, Proc. SPIE, 2552, 33

Ashley, M. C. B., Burton, M. G., Storey, J. W. V., Lloyd., J. P., Bally, J., Briggs, J. W., \& Harper, D. A. 1996, PASP, 108, 721

Burton, M. G., Storey, J. W. V., \& Ashley, M. C. B. 2001, PASA, 18,158

Burton, M. G., et al. 1994, PASA, 11, 127

Chamberlain, M. A., Ashley, M. C. B., Burton, M. G., Phillips, A. Storey, J. W. V., \& Harper, D. A. 2000, ApJ, 535, 501

CMDL 2001, Climate Monitoring \& Diagnostics Laboratory, http://www.cmdl.noaa.gov/met/

Hereld, M. 1994, ExA, 3, 87

Hoffman, W., Lemke, D., \& Thum, C. 1977, ApOpt, 16, 3125

Jacobson, M. Z. 1999, Fundamentals of Atmospheric Modeling (Cambridge: Cambridge Univeristy Press)

Lang, K. R. 1980, Astrophysical Formulae (New York: SpringerVerlag)

Leinhert, C., et al. 1998, A\&A Suppl. Series, 127, 1
Mandolesi, N., et al. 1998, A\&A, 331, 463

Matsumoto, T., Akiba, M., \& Murakami, H. 1988, ApJ, 332, 575

Matsumoto, T., Matsuura, S., \& Noda, M. 1994, PASP, 106, 1217

Matsumoto, T., Kawada, M., Murakami, H., Noda, M., Matsuura, S., Tanaka, M., \& Narita, K. 1996, PASJ, 48, L47

Matsuura, S., Kawada, M., Matsuhara, H., Matsumoto, T., Noda, M., Tanaka, M., \& Bock, J.J. 1994, PASP, 106, 770

McKewon, J. M., \& Phillips, L.F. 1975, Chemistry of the Atmosphere (London: Edward Arnold)

Nguyen, H. T., Rauscher, B. J., Severson, S. A., Hereld, M., Harper, D. A., Loewenstein, R. F., Mrozek, F., \& Pernic, R. J. 1996, PASP, 108, 718

Noda, M., Chritov, V. V., Matsuhara, H., Matsumoto, T., Noguchi, K., Sato, S., \& Murakami, H. 1992, ApJ, 391, 456

Phillips, A., Burton, M. G., Ashley, M. C. B., Storey, J. W. V., Lloyd, J. P., Harper, D. A., \& Bally, J. 1999, ApJ, 527, 1009

Rothman, L. S., et al. 1998, JQSRT, 60, 665

Smith, C. H., \& Harper, D. A. 1998, PASP, 110, 747

Storey, J. W. V. 1998, in Astrophysics from Antarctica, ASP Conf. Series 141, ed. G. Novak \& R. H. Landsberg (San Fransisco: ASP), 313

Storey, J. W. V., Ashley, M. C. B., \& Burton, M. G. 1996, PASA, 13,35

Storey, J. W. V., Ashley, M. C. B., Boccas, M., Phillips, M. A., \& Schinckel, A. E. T. 1999, PASP, 760, 765

Townes, C. H., \& Melnick, G. 1990, PASP, 102, 357

Valenziano, L., Cavaliere, F., \& Miriametro, A. 1997, ExA, 7,421 